Trauma Berufskrankh 2010 - 12 [Suppl 4]:434-438 DOI 10.1007/s10039-010-1642-7

Online publiziert: 15. Juli 2010

(c) Springer-Verlag 2010

\author{
M. Faschingbauer \\ BG-Unfallkrankenhaus Hamburg
}

\title{
Man lernt nie aus! ... auch nicht das Implantat
}

setzt. Auf Basis der hiermit erhaltenen Erfahrungen wurde in Zusammenarbeit mit der Technischen Universität Hamburg-Harburg eine Kombination aus der millionenfach bewährten Elektronik und der winkelstabilen Platte entwickelt:

Eine winkelstabile Platte wurde mit einem Dehnungsmessstreifen und einem Transponder verkapselt (• Abb. 1). Mit Hilfe eines Lesegeräts kann eine axiale Belastung dieser elektronisch instrumentierten Platte gemessen werden.

\section{Einsatz des intelligenten Implantats}

\section{Anwendungsgebiet}

\section{Entwicklung des intelligenten Implantats}

Im Berufsgenossenschaftlichen Unfallkrankenhaus (BG-Unfallkrankenhaus) Hamburg wurden bereits über 4000 winkelstabile Implantate an den unterschiedlichsten anatomischen Regionen einge-
Nach entsprechenden Labor- und Tierversuchen wurde das System in die Klinik eingeführt, mit der Absicht, diese Platte primär bei Oberschenkelpseudarthrosen einzusetzen. Gerade vor dem Hintergrund, dass bis zu 12,5\% aller Femurfrak-

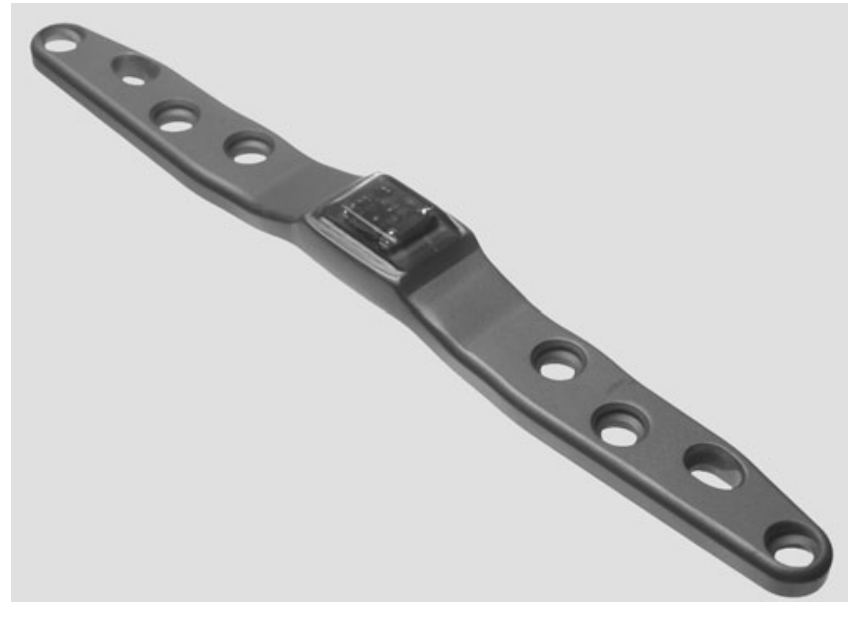

Abb. 14 Femurfixateur interne mit Telemetrieelektronik turen nach einer Marknagelung in einer Pseudarthrose enden [1], wurde dieses intelligente Implantat bei Patienten mit Oberschenkelpseudarthrosen eingesetzt, welche bereits mehrfache, fehlgeschlagene Revisionsoperationen erlitten hatten.

\section{Operatives Vorgehen}

Das standardisierte Vorgehen im BG-Unfallkrankenhaus Hamburg lässt sich wie folgt beschreiben: Nach Entfernung des einliegenden Materials und anschließender Pseudarthrosenrevision mit Anfrischung und Eröffnung des Markraums nach proximal und distal wird die Pseudarthrose mit der elektronisch armierten Platte stabilisiert. Dabei werden stets eine autologe Spongiosaplastik durchgeführt und, da in einer hausinternen Studie bei $40 \%$ der Oberschenkelpseudarthrosen entweder intraoperativ ein positiver Keimnachweis zu verzeichnen oder in der obligat entnommenen Histologie eine Osteitis nachweisbar war, zusätzlich eine PMMA-Kette (PMMA: Polymethylmethacrylat) eingelegt.

\section{Nachbehandlung/ Messwerterhebung}

Postoperativ werden die Patienten in definierten Abständen klinisch und radiologisch kontrolliert. Dabei wird über einen Lastaufnehmer eine definierte axiale Last eingebracht und gleichzeitig unblutig mit dem Lesegerät die Belastung des Implantates gemessen, und zwar sowohl in stehender als auch in liegender Position. Zu- 
sätzlich erfolgt eine Belastung im Varusund Valgusstress.

Trägt man die externe Kraft gegen das Biegemoment auf, ergibt sich eine lineare Gerade, welche die Elastizität darstellt. Je steiler diese Gerade ist, umso höher ist die Elastizität.

\section{Ergebnisse}

Im Folgenden wird der Heilungsverlauf von 2 Patienten demonstriert.

Fall 1. Der 46 Jahre alte Patient hatte sich eine Oberschenkelschaftfraktur zugezogen, welche mittels unaufgebohrtem Marknagel operativ versorgt worden war. Bei ausbleibender Bruchheilung war eine Revisionsoperation mit Spongiosaplastik und einem Nagelwechsel vorgenommen worden. Bei weiterhin ausbleibender Heilung wurde im BG-Unfallkrankenhaus Hamburg die Versorgung mit dem winkelstabilen, elektronisch instrumentierten Implantat in beschriebener Weise durchgeführt.

Nach 5 Wochen war bereits ein Abfall der Belastung im Implantat auf 30\% des Ausgangswertes zu verzeichnen, obwohl radiologisch keinerlei Durchbauungsvorgänge festzustellen waren. Aufgrund der gemessenen Daten erfolgte eine Belastungssteigerung. Nach 9 Wochen waren nur noch $20 \%$ des Ausgangswertes bei Belastung im Implantat zu messen - bei radiologisch weiterhin fehlender knöcherner Durchbauung. Dennoch wurde Vollbelastung erlaubt. Nach 16 Wochen zeigte sich bei einem Rückgang der Implantatbelastung auf fast o auch schließlich eine radiologisch nachweisbare knöcherne Durchbauung.

Fall 2. Beim dem 55-jährigen Patienten war eine subtrochantäre Oberschenkelfraktur primär mit einem $\gamma$-Nagel versorgt worden. Bei ausbleibender Heilung wurde ein Revisionseingriff mit Spongiosaplastik und Einsatz einer zusätzlichen lateralen Platte vorgenommen, worauf es zwar zu einer knöchernen Konsolidierung kam, jedoch mit einer Fehlstellung mit $30^{\circ}$ Außendrehung und $10^{\circ} \mathrm{Va}$ rus. Deshalb wurde subtrochantär eine Korrekturosteotomie vorgenommen, die Stabilisierung erfolgte mit einem winkel-

Trauma Berufskrankh 2010 · 12 [Suppl 4]:434-438 DOI 10.1007/s10039-010-1642-7

C) Springer-Verlag 2010

\section{Faschingbauer \\ Man lernt nie aus! ... auch nicht das Implantat}

\section{Zusammenfassung}

Im BG-Unfallkrankenhaus Hamburg wurde in

Zusammenarbeit mit der Technischen Universität Hamburg-Harburg ein als intelligentes Implantat bezeichnetes Fixateur-interne-System entwickelt. Durch das Aufbringen einer modernen Mikroelektronik auf eine Platte ist es möglich, deren Belastung teleme-

trisch zu messen. Das System wurde zunächst an Kunststoffmodellen, anschließend im Tierversuch geprüft. Klinisch wurde das neue Implantat bisher bei 27 Patienten mit Oberschenkelpseudarthrose eingesetzt. Die ersten Ergebnisse zeigen eine gute Korrelation zwischen der knöchernen Heilung und dem Verlauf der empfangenen Signale. Der Einsatz dieses Systems erlaubt eine kontrollier- te Nachbehandlung von Osteosynthesen mit optimaler Ausnutzung der Belastungsmöglichkeit der Extremität. Die Stabilität des Kallus kann direkt gemessen und muss nicht indirekt über ein Röntgenbild interpretiert werden. Zudem könnten Probleme in der Frakturheilung frühzeitig erkannt und ihnen entsprechend begegnet werden. Intelligente Implantate werden u. E. für die Osteosynthese der Zukunft wichtige Werkzeuge darstellen.

\section{Schlüsselwörter}

Fixateur-interne-System · Intelligentes Implantat - Telemetrische Messung .

Tierversuch · Pseudarthrosen

\section{You never stop learning! Nor does the implant}

\section{Abstract}

At the Trauma Centre Hamburg, together with the University of Technology Hamburg, an internal fixation system, known as the "intelligent implant", has been developed. By applying a modern microelectronic circuit onto a plate, it is possible to measure its load telemetrically. The system was tested in vitro and in an animal study. The new implant was applied in 27 patients with a nonunion in a clinical setting. Initial results in patients with femoral non-unions showed a good correlation between bone healing and the course of the received signals. Using the system, a con- trolled follow-up of osteosyntheses with optimal weight bearing is possible. We can now directly measure callus stability instead of estimating it by means of radiograph interpretation. Additionally, problems during fracture healing can be recognized early and treated accordingly. Intelligent implants will be important tools for osteosynthesis in the future.

\section{Keywords}

Internal fixation system · Intelligent implant · Telemetric measurement · Animal study · Non-unions 


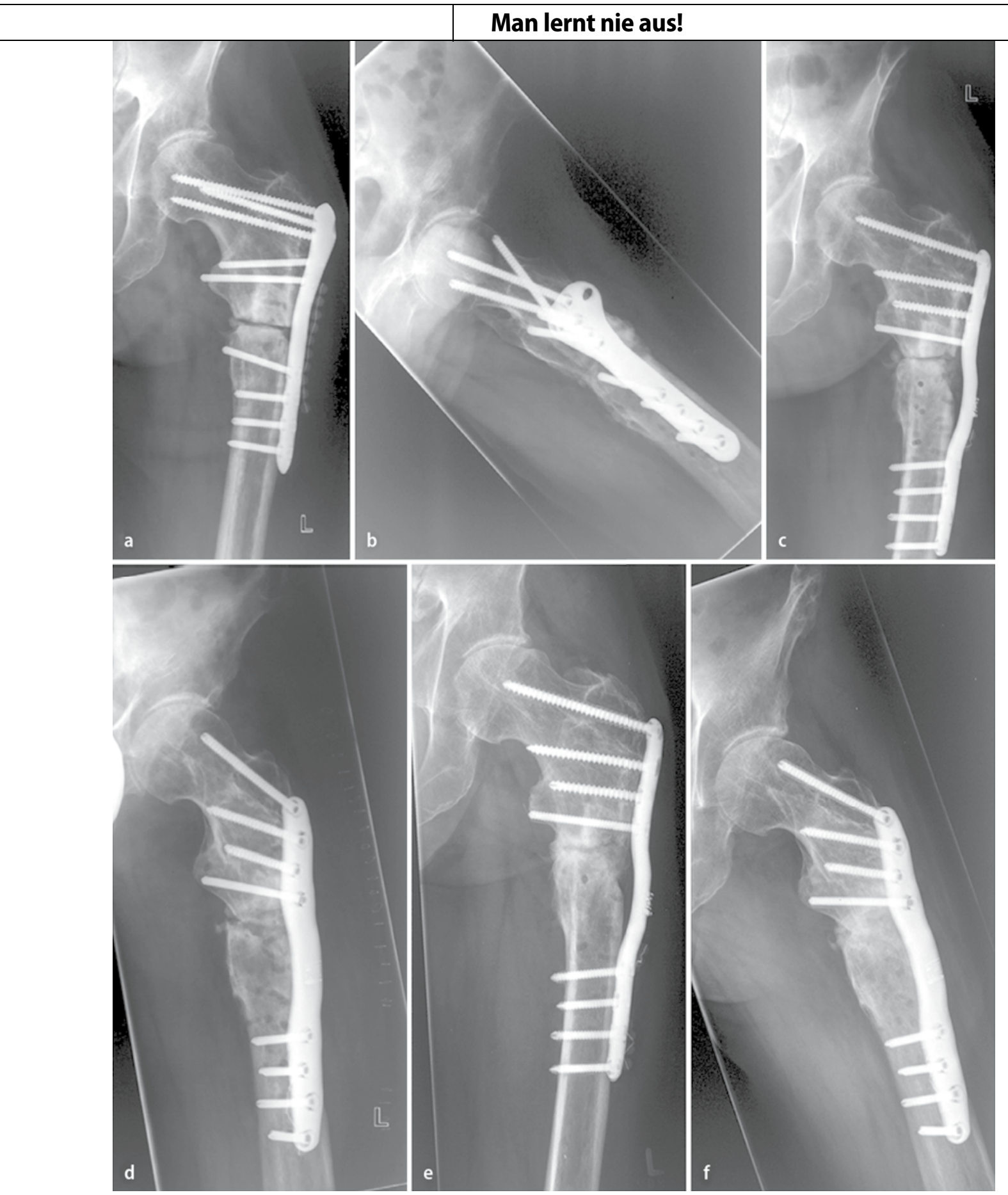

Abb. $2 \varangle$ Fallbeispiel 2, 55-jähriger Patient mit Pseudarthrose am proximalen Femur, a, b Röntgenbefund präoperativ, c, d Versorgung mit dem intelligenten Implantat, e, f Konsolidierung nach 34 Wochen

stabilen Implantat sowie einer Spongiosaplastik (- Abb. 2a,b).

Bei ausbleibender Heilung wurde wiederum das standardisierte Vorgehen mit dem intelligenten Implantat im BG-Unfallkrankenhaus Hamburg durchgeführt. Im weiteren Verlauf zeigte sich eine protrahierte knöcherne Bruchheilung, wobei nach etwa 13 Wochen eine Reduzierung der Belastung im Implantat auf knapp 35\% $\mathrm{zu}$ verzeichnen war, bei radiologisch weiterhin bestehendem deutlichem Pseudarthrosenspalt. Auch nach 19 Wochen war radiologisch keine knöcherne Durchbauung darzustellen ( $\mathbf{O}$ Abb. 2c,d), aber die Belastung im Implantat war auf 25\% zurückgegangen (- Abb. 3). Es erfolgte Vollbelastung, worunter nach 34 Wochen auch radiologisch eine knöcherne Konsolidierung nachzuweisen war (- Abb. 2e,f).

Am BG-Unfallkrankenhaus Hamburg wurden zwischenzeitlich 27 Patienten mit diesem Verfahren operiert. Dabei handelte es sich größtenteils um $\mathrm{Pa}$ tienten mit atrophen und oligotrophen
Pseudarthrosen (18/5), die überwiegend im mittleren und distalen Drittel lokalisiert waren (15/7). Das Durchschnittsalter lag bei 38 Jahren, die Geschlechterverteilung männlich:weiblich betrug 23:4 (• Abb. 4).

Die Dauer der Vorbehandlung belief sich im Durchschnitt auf 15,2 Monate und reichte von 5-69 Monaten (• Abb. 5).

Es waren durchschnittlich 2,26 Operationen vorausgegangen, wobei es sich in den meisten Fällen um eine Versorgung 
mit unaufgebohrten Marknägeln handelte (- Abb. 6).

Unter den 27 operierten Patienten kam es bei 22 Pseudarthrosen durch eine einzige Operation zur stabilen Durchbauung. Bei 3 Patienten ist die Behandlung noch nicht abgeschlossen, wobei sowohl die Kurvenwerte als auch die Röntgenbefunde einen positiven Verlauf erwarten lassen. Ein Verfahrenswechsel war 2-mal erforderlich.

\section{Komplikationen}

Fall 1. Bei der 49-jährigen Patientin mit einem Körpergewicht von $85 \mathrm{~kg}$ bei einer Körpergröße von etwa $150 \mathrm{~cm}$ war eine proximale Oberschenkelfraktur mit einem langen $\gamma$-Nagel operativ versorgt worden. Bei ausbleibender Knochenbruchheilung erfolgte das standardisierte Vorgehen im BG-Unfallkrankenhaus Hamburg. Bereits nach 3 Wochen stellte sich die Patientin mit einem Plattenbruch vor, der durch Reosteosynthese mit einem Druckplattenfixateur versorgt wurde, da die Patientin eine weitere Operation mit dem intelligenten Implantat nicht wünschte.

Die Platte wurde in Biomechaniklabor untersucht, und die Bruchenden sprachen für einen spröden Gewaltbruch.

Fall 2. Dieser Patient hatte sich ebenfalls eine Oberschenkelschaftfraktur zugezogen, welche mittels Marknagel operativ versorgt worden war. Bei ausbleibender Bruchheilung erfolgte die Versorgung mit dem intelligenten Implantat. Der sportliche junge Mann stürzte im weiteren Verlauf, und es kam zu einer deutlichen Verbiegung der einliegenden Platte. Ein Ausriss der Schrauben oder eine Schraubenlockerung ließen sich nicht nachweisen. Aufgrund der durch den Sturz entstandenen Varusverbiegung am Oberschenkel wurde eine Reosteosynthese mit einem Druckplattenfixateur (Wunsch des $\mathrm{Pa}$ tienten) durchgeführt.

Im Labor wurde versucht, die beschriebene Situation nachzustellen, um ermessen zu können, bei welcher axialen Belastung es zu einer Verbiegung der Platte kommt. Um eine entsprechende plastische Verformung der Platte zu bewerkstelligen, mussten experimentell $2000 \mathrm{~N}$

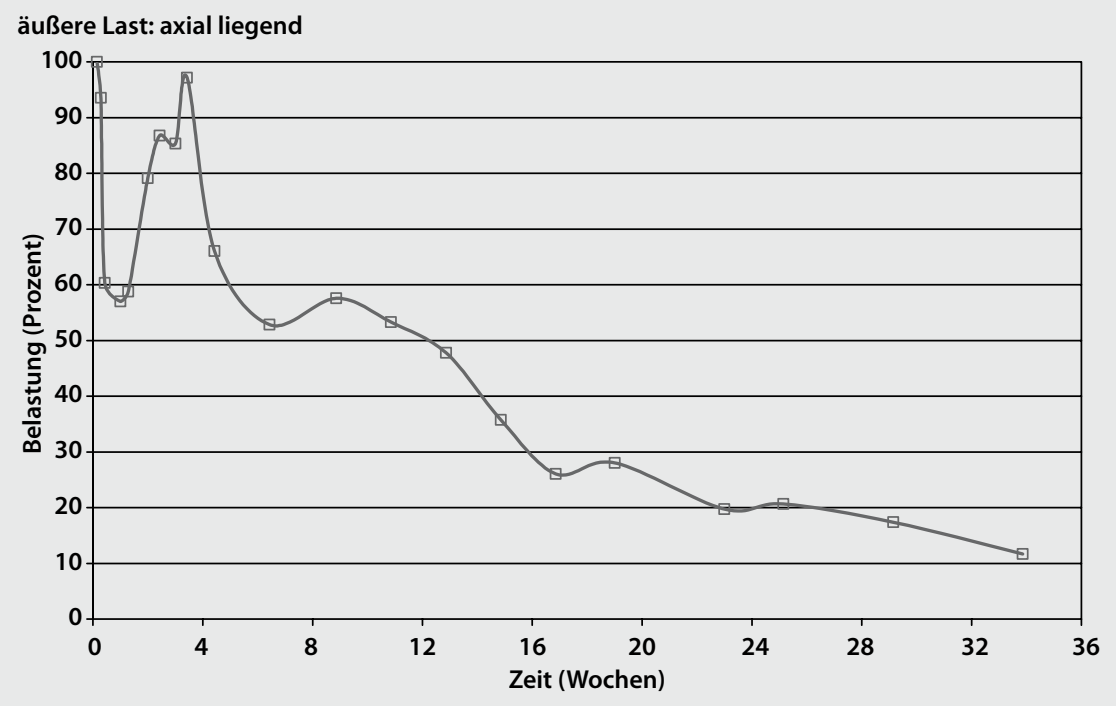

Abb. $3 \Delta$ Fallbeispiel 2, 55-jähriger Patient mit Pseudarthrose am proximalen Femur, Abnahme der telemetrisch bestimmten Elastizität der Osteosynthese durch die zunehmende Kallussteifigkeit

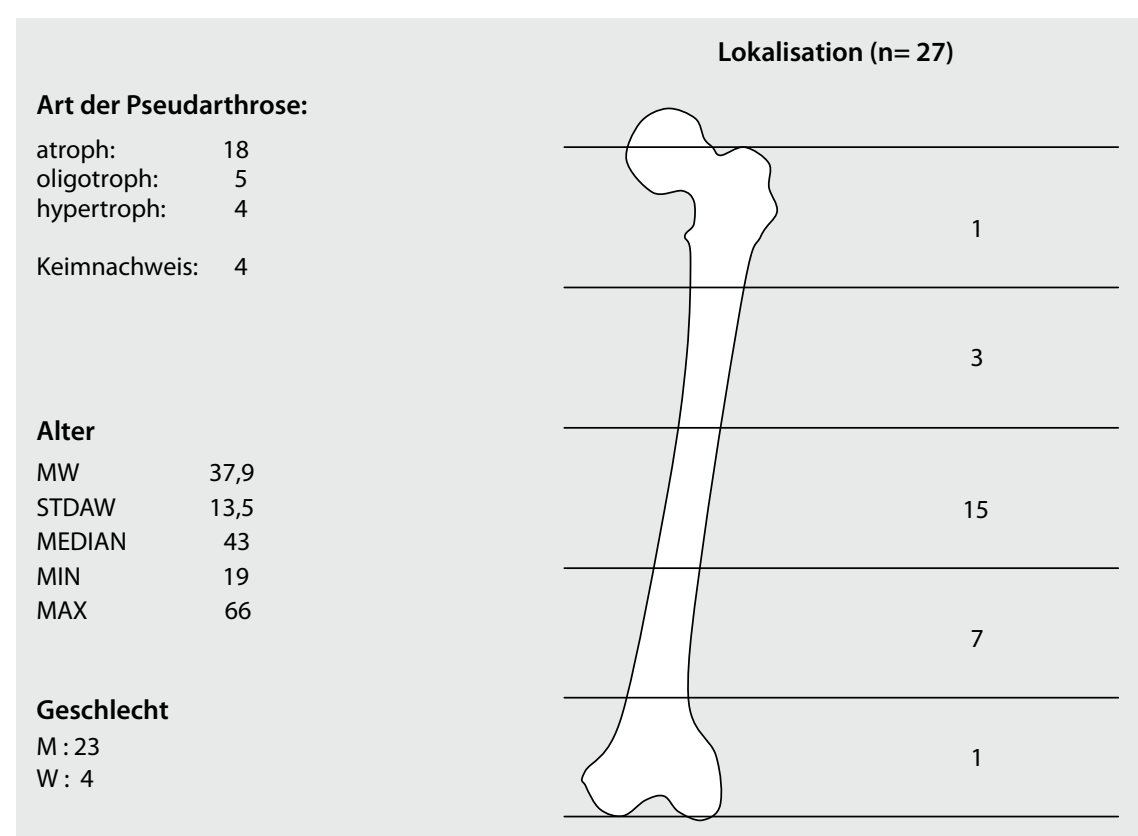

Abb. $4 \Delta$ Patientenkollektiv, MAX Maximum, MIN Minimum, MW Mittelwert, STDAW Standardabweichung

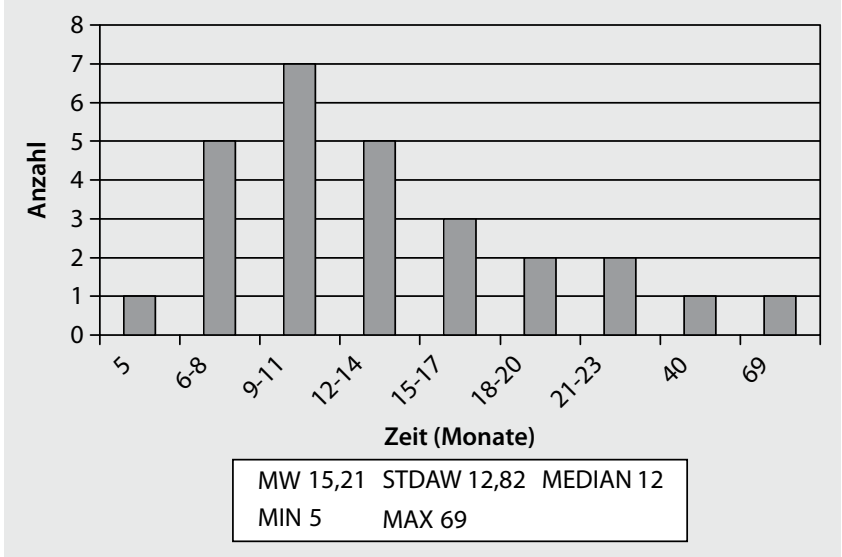




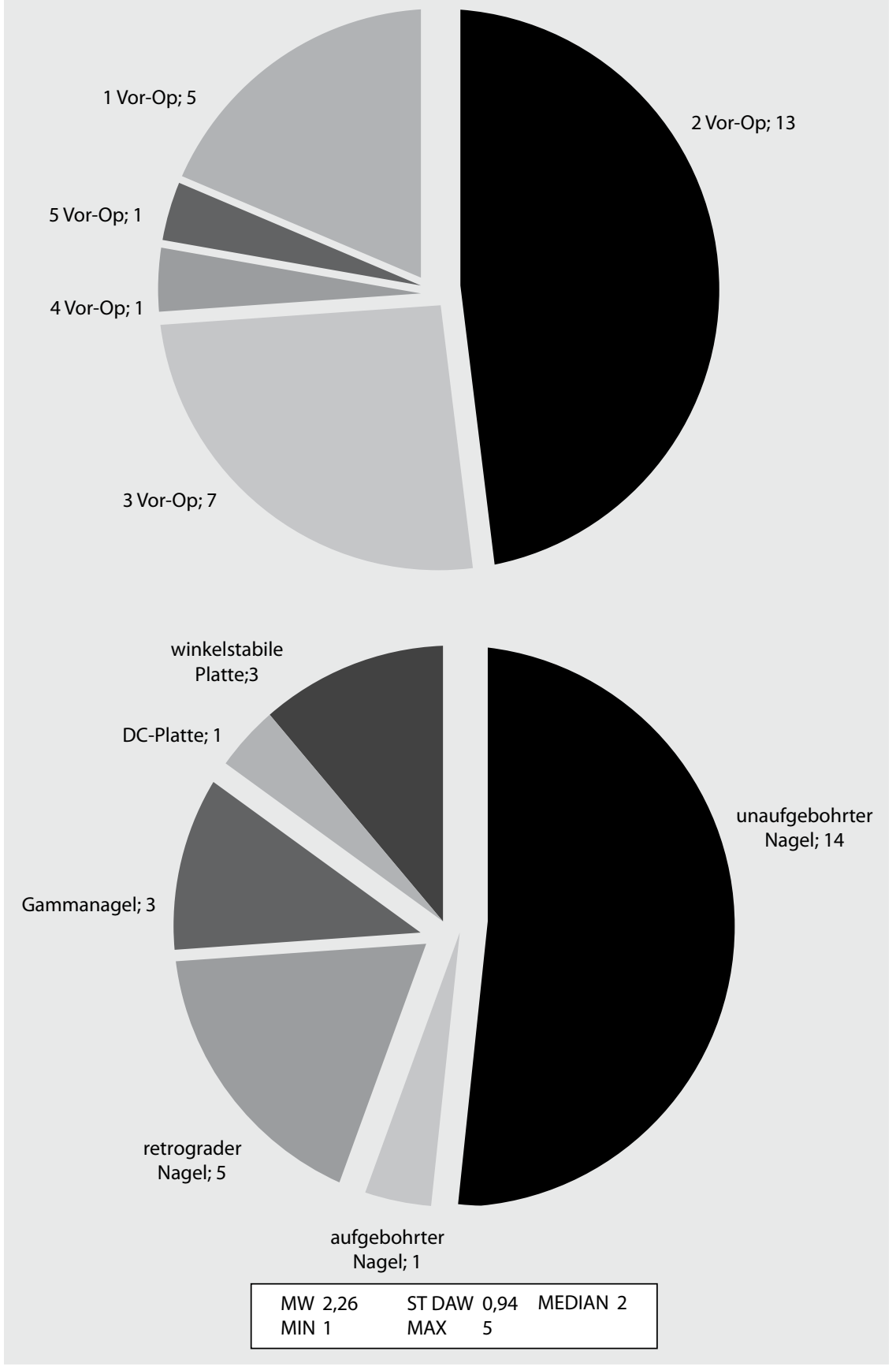

Abb. $6 \Delta$ Voroperationen ( $\mathrm{n}=27), D C_{\prime \prime}$ dynamic compression", MAX Maximum, MIN Minimum, MW Mittelwert, STDAW Standardabweichung, Vor.-OPVoroperation

angewendet werden. Bedenkt man, dass bei der primären Osteosynthese des Oberschenkels eine mediale knöcherne Abstützung bestand, aber im Laborversuch eine künstliche Defektstrecke von $2 \mathrm{~cm}$ gewählt wurde, bedeutet dies, dass bei zusätzlicher medialer Abstützung eine noch höhere axiale Belastung als $2000 \mathrm{~N}$ erforderlich ist, damit es zu einer Verbiegung der Platte kommt.

\section{Fazit für die Praxis}

Aus den bisher mit dem intelligenten Implantat versorgten Fällen von Oberschenkelpseudarthrosen wurden viele Erkenntnisse gezogen: Mit diesem System können erstmals die Frakturheilung direkt beurteilt sowie die Stabilität des Kallus gemessen und nicht indirekt über ein Röntgenbild interpretiert werden. Damit ist es möglich, die Nachbehandlung zu optimieren, in dem Überlastun- gen vermieden werden und eine individuelle Belastungssteigerung genau gesteuert werden kann.

\section{Korrespondenzadresse}

Dr. M. Faschingbauer

BG-Unfallkrankenhaus Hamburg,

Bergedorfer Straße 10, 21033 Hamburg

M.Faschingbauer@buk-hamburg.de

Interessenkonflikt. Keine Angaben

\section{Literatur}

1. Roetman B, Scholz N, Muhr G, Möllenhoff G (2008) Die additive Plattenosteosynthese bei Femurpseudarthrosen nach intramedullärer Stabilisierung Strategie nach gescheiterter Marknagelung am Femur. Z Orthop Unfall 146(5):586-590 